\title{
Laser-Induced Superhydrophobic Ti-Ni Shape Memory Alloy with Corrosion Resistance and Self-Cleaning Properties
}

\author{
Tianchi Chen ${ }^{1}$, Shirong $\mathrm{Ge}^{1}$ and Hongtao $\mathrm{Liu}^{2 *}$ \\ ${ }^{1}$ School of Mechanical \& Electrical Engineering, \\ ${ }^{2}$ School of Materials Science and Engineering, China University of Mining and Technology, Xu- \\ zhou 221116, People's Republic of China \\ *E-mail:Liuht100@126.com
}

\begin{abstract}
Owing to the unique water repellence and self-cleaning properties, superhydrophobic surface endows them with greatly enhanced anti-adhesion. Thus, a facile and controllable laser direct writing approach is employed to fabricate a superhydrophobic surface on a Titanium nickel (Ti-Ni) shape memory alloy substrate as barrier against blood lipid deposition. The wettability and morphology of the consequent superhydrophobic surface are characterized by contact angle (CA), scanning electron microscopy (SEM). The unique trench textures composed of fish scale-like microstructure on the bottom and micro/nano-particles on the ridge are successfully obtained by controllable laser fluence and scanning speed. After $1 \mathrm{H}, 1 \mathrm{H}, 2 \mathrm{H}, 2 \mathrm{H}$-perfluorodecyltriisopropoxysilane modification, this unique trench textures surface shows excellent superhydrophobicity with calf serum contact angles (CA) of $156^{\circ}$ and sliding angles (SA) less than $5^{\circ}$. Meanwhile, the surface shows oleophobicity with glycerol contact angles (CA) of $130^{\circ}$ and sliding angles (SA) of $30^{\circ}$. The anticontamination and anticorrosion behaviors of the as-prepared superhydrophobic surface are investigated by self-cleaning test, potentiodynamic polarization. The potentiodynamic polarization results show that the as-prepared superhydrophobic surface can greatly enhance corrosion resistance, and the self-cleaning test results indicate that the as-prepared superhydrophobic surface is able to remove the dust under water flushing.
\end{abstract}

DOI: 10.2961/jlmn.2017.03.0007

Keywords: Ti-Ni shape memory alloy, laser direct writing, superhydrophobicity, oleophobicity, self-cleaning

\section{Introduction}

Inspired by lotus leaf [1], butterfly wing [2], salvinia leaf [3] and water-strider legs [4], superhydrophobic surface, with the water contact angle above $150^{\circ}$ and the sliding angle less than $5^{\circ}$, has arouse more and more interest in potential applications, such as self-cleaning, anticorrosion, anti-icing and drag reduction. Many researchers investigated that low surface energy and hierarchical micro/nano structures are two main factors to achieve superhydrophobic surface. Many methods have been investigated to achieve hierarchical micro/nano-structures, such as electrochemical methods [5], Sol-Gel methods [6], spray methods [7] and chemical vapor deposition [8]. Compared to the above-mentioned method, laser direct writing technology is employed as a promising method for surface texturing because of its structure controllability, non-pollution and localization manufacture. Moreover, the micro/nano structures generated by laser direct writing are original structures without consideration of the adhesion between coating and substrate. Thus, laser direct writing can be seen as an ideal method to fabricate superhydrophobic surface [9, 10]. Hong [11] fabricated hybrid scale micro/nanostructures on the carbon nanotube (CNT) surface by femtosecond laser micro-machining and chemical vapor deposition to realize superhydrophobicity.

Ti-Ni memory alloy is the most common used memory alloy in electronics, machinery, energy and medical fields with high strength, good corrosion resistance and good biocompatibility. Thus, Ti-Ni memory alloy is widely used as heart stents. Some problems still remain when the stent are implanted into the blood vessel for the long time, such as the heart stent would be covered by blood lipid, leading to the heart stent failure. Therefore, it is necessary to enhance their blood and lipid repellence on the stent's surface in blood media, which will greatly extend stent's lifetime. However, few study reports to construct superhydrophobic surface on the Ti-Ni surface to improve its blood or lipid repellence.

In this study, we used an ultraviolet nano-second laser, which is compact, cost-effective compared to the femtosecond laser, to fabricate superhydrophobic Ti-Ni memory alloy surface. The relationship between surface wettability and morphology are investigated. We also test its anticontamination performance. The anticorrosion behaviors of the surface are studied by potentiodynamic polarization. In addition, this work provides that superhydrophobic Ti-Ni alloy surface has a potential application in preventing blood lipid deposition on the implant surface.

\section{Experiment methods}

Ti-Ni memory alloy with a size of $20 \times 20 \mathrm{~mm}$ and thickness of $1 \mathrm{~mm}$ are purchased from Kelloggs Research Labs. The sample is mechanically polished, then rinsed with deionized water, degreased by a $30 \mathrm{~min}$ ultrasonic bath in acetone, dried in a dry box. FAS- $17(1 \mathrm{H}, 1 \mathrm{H}, 2 \mathrm{H}$, 
2H-perfluorodecyltriisopropoxysilane) (97\%) is purchased from SICONG chemical.

The Ti-Ni alloy surface are mounted on the machine table. A 25 ns ultraviolet laser has a working wavelength of $355 \mathrm{~nm}$, repetition rate of $6.8 \mathrm{kHz}$. The focused laser beam is controlled by a laser marker with a focus lens ( $\mathrm{f}=160 \mathrm{~mm}$ ) to move predetermine direction. The radius of beam waist of the laser pulses after the focusing lens was approximately $18 \mu \mathrm{m}$. The Ti-Ni alloy sample is raster scanned at the different speeds $(\mathrm{V}=10 \mathrm{~mm} / \mathrm{s} \sim 80 \mathrm{~mm} / \mathrm{s})$ with the overlapping spacing $\Delta=40 \mu \mathrm{m}$. The cumulative laser fluence is changed from $3.5 \mathrm{~J} / \mathrm{cm}^{2}$ to $28 \mathrm{~J} / \mathrm{cm}^{2}$. After laser processing, samples are cleaned with distilled water and dried at $60{ }^{\circ} \mathrm{C}$ for $1 \mathrm{~h}$. Finally, the samples are immersed into 2 wt $\%$ FAS-17 $1 \mathrm{H}, \quad 1 \mathrm{H}, \quad 2 \mathrm{H}, \quad 2 \mathrm{H}-$ perfluorodecyltriisopropoxysilane) ethanol solution for $1 \mathrm{~h}$ followed by washing with ethanol and drying in an oven at $100{ }^{\circ} \mathrm{C}$ for $1 \mathrm{~h}$.

Surface morphologies are measured using a scanning electron microscope (FEI limited, Quanta 250). JC2000D2A contact angle measuring device is used to measure wettability. The water contact angle (CA) and sliding angle are measured with $5 \mu \mathrm{L}$ calf serum and $10 \mu \mathrm{L}$ glycerol droplet dripped on the five different points for each surface.

The corrosion resistance of superhydrophobic surface are carried out in electrochemical corrosion test. The sample is immersed into $20 \mathrm{wt} \%$ calf serum corrosion aqueous solutions on an electrochemical workstation (RST5000) in a three-electrode system. The superhydrophobic Ti-Ni alloy surface, a platinum plate and saturated calomel electrode (SCE) are used as the working electrode, counter and reference electrode, respectively.

\section{Results and discussion}

Fig. 1 shows the morphology of Ti-Ni alloy surface in different laser powers from $3.5 \mathrm{~J} / \mathrm{cm}^{2}$ to $28 \mathrm{~J} / \mathrm{cm}^{2}$, at the fixed scanning speed of $50 \mathrm{~mm} / \mathrm{s}$. Fig. 2 demonstrates the contact angle and sliding angle of sample processed with corresponding laser fluence from $3.5 \mathrm{~J} / \mathrm{cm}^{2}$ to $28 \mathrm{~J} / \mathrm{cm}^{2}$ at the same scanning speed of $50 \mathrm{~mm} / \mathrm{s}$. When the laser fluence is $3.5 \mathrm{~J} / \mathrm{cm}^{2}$, the surface remains flat. There are only some re-melting microprotrusions randomly distributing among the scanning path, as shown in Fig.1 (a). However, this kind morphology cannot achieve a superhydrophobic surface with a calf serum contact angle of $136^{\circ}$, and its sliding angle as high as $38^{\circ}$. The calf serum is stuck at the Ti-Ni alloy surface, which could be ascribed to the lack of rough micro/nano-composite structure. With the laser fluence increasing to $16 \mathrm{~J} / \mathrm{cm}^{2}$, the trench texture with the period of $40 \mu \mathrm{m}$ can be clearly seen on the surface. Many fish scale-like microstructures covers the trench bottom, the boundary among two trenches remain flat, as shown in Fig.1 (b). The contact angle and sliding angle of fish scalelike microstructures surface are about $143^{\circ}$ and $20^{\circ}$, respectively. This is due to the fact that trench textures can increase surface roughness, which can lead to contact angle increasing. However, only trench textures covered with fish scale-like microstructures cannot obtain superhydrophobic surface. As the laser fluence further increases to $20 \mathrm{~J} / \mathrm{cm}^{2}$, the trench becomes deep and fish scale-like textures are more visible, as shown in Fig, 1(c). Moreover, the edge of the trench becomes rough and some micro-particles rarely accumulate and finally form ridges. The contact angle and sliding angle of rough ridge textures combined with fish scale-like microstructures surface are about $150^{\circ}$ and $8^{\circ}$, respectively. However, the superhydrophocity of Ti-Ni surface still remains unstable and superhydrophobic state can be failed for the long time contact with water for several hours. This is due to the height between ridge and trench bottom is not sufficient to support the water droplet for a long time. When the laser fluence increases to $28 \mathrm{~J} / \mathrm{cm}^{2}$, the ridge becomes wide. Some micro-particles and hair-like nanostructures cover all the ridge part. The fish scale-like textures are more obvious. The trench deepens with laser fluence. Accompanying with the formation of this unique rough hierarchical micro/nano structures on the ridge and fish scale-like structure at the bottom, the as-prepared surface achieves a stable superhydrophobicity with contact angle and sliding angle of $156^{\circ}$ and $2^{\circ}$. Water droplets are hardly to stay on this surface and rolling around. In addition, the contact angle and sliding angle of a $10 \mu \mathrm{L}$ glycerol are measured and found to be $150^{\circ}$ and $15^{\circ}$, respectively, which have achieved approximately superoleophobic state. This could be attributed to the reasonable micro/nanohierarchical structures on the ridge and the low energy material of FAS-17 grafted on as-prepared Ti-Ni surface. Hence, it is very important to achieve micro/nanohierarchical structures on the ridge and fish scale-like structure at the bottom. From the SEM images, we find that the laser fluence does not mainly influence the formation of fish scale-like microstructures at the bottom of trench. The laser fluence can control the roughness of ridge. The schematic diagram of hierarchical micro/nano structures forming on the ridge part can be seen in Fig. 2. The main material removal are melting and evaporation of metal surface at the low laser fluence because of it cannot reach the fragmentation ablation threshold [12]. The ablated area is to be smooth, the ablated trench height (H1) is the lowest, as shown in Fig.3 (a). With the laser fluence increasing, when the surface temperature increase to some breakpoint, a large amount of vaporization nucleus appear under the melting materials surface. When the steam pressure on the melting material is greater than surface tension of melting liquid, the melting materials begin to ejecting away from center of laser beam [13]. Meanwhile, steam pressure results in the melting area to be pushed to two sides and the accumulated re-melting layer forms ridge with two peaks. Moreover, Electrons around the irradiated region easily form plasma in the high laser fluence $[14,15]$. The plasma would interfere the energy absorption of the ablated surface and make the splashing droplet re-deposited on the ridge region [16], as shown in Fig. 3 (b). Finally, when the laser fluence increases to some value, the phase explosion would continuously happen on the Ti-Ni surface. Thus, the ridge part is covered with some micro-particles and hair-like nanostructures and the laser ablated deepest trench (H3), as shown in Fig. 3 (c). 


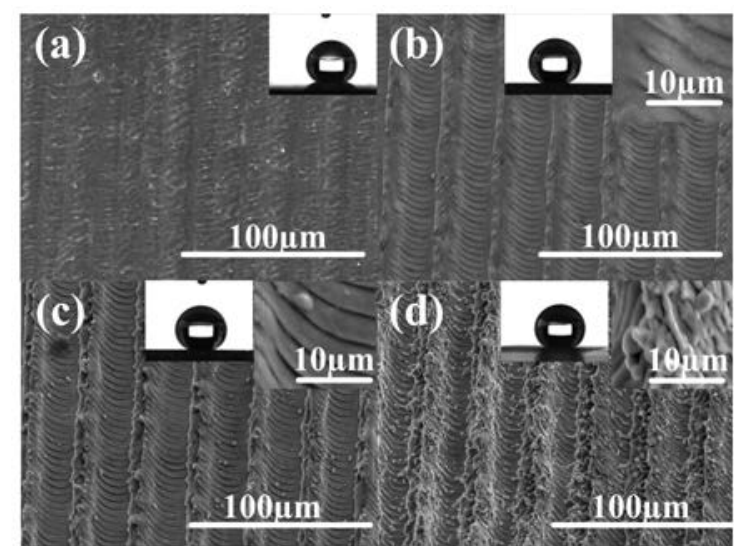

Fig. 1 SEM images of the Ti-Ni memory alloy surface under different laser fluence, (a) $3.5 \mathrm{~J} / \mathrm{cm}^{2}$, (b) $16 \mathrm{~J} / \mathrm{cm}^{2}$, (c) $20 \mathrm{~J} / \mathrm{cm}^{2}$, (d) $28 \mathrm{~J} / \mathrm{cm}^{2}$.

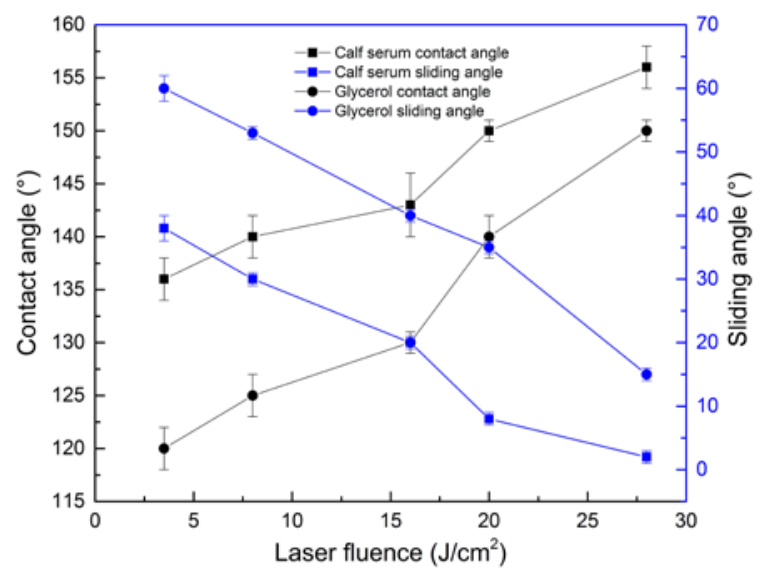

Fig. 2 Contact angles and sliding angles of Ti-Ni alloy surface under different laser fluences.

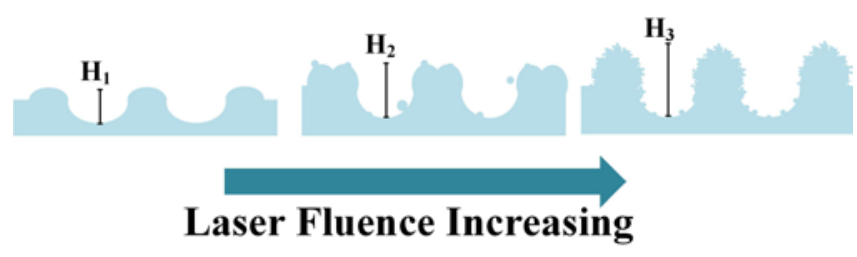

Fig. 3 Morphology evolution of the hierarchical micronanostructures with laser fluence

In order to further investigate the formation mechanism of fish scale-like microstructures on the bottom of trench, we processed surfaces with the scanning speed changing from $10 \mathrm{~mm} / \mathrm{s}$ to $80 \mathrm{~mm} / \mathrm{s}$ at the fixed laser fluence of 20 $\mathrm{J} / \mathrm{cm}^{2}$, as shown in Fig. 4. At the relative low speed of 10 $\mathrm{mm} / \mathrm{s}$, the trench texture disappears, many random distributed microprotrusions consist of some staggered nanoparticles. The higher-magnification image of Fig. 4(a) demonstrates that the rough Ti-Ni surface is composed of numerous irregular spherical nanoparticle intercrossed on the microprotrusions and seem perpendicular to the Ti-Ni surface. As the scanning speed increases to $30 \mathrm{~mm} / \mathrm{s}$ (Fig. 4 (b)), trench textures cover the whole surface. The fish scale-like microstructure is not obvious at the bottom of trench. The trench bottom and side wall are covered with many nanoparticles. When the scanning speed increases to $50 \mathrm{~mm} / \mathrm{s}$, the fish scale-like microstructure is obviously seen at the bottom of trench. The distance between two scales is about $7 \mu \mathrm{m}$, as shown in Fig. 4 (c). Serrated strip textures covered with some irregular nanoparticles are shown in Fig. 4 (d). Thus, fish scale-like microstructures can be obtained by adjusting the scanning speed.

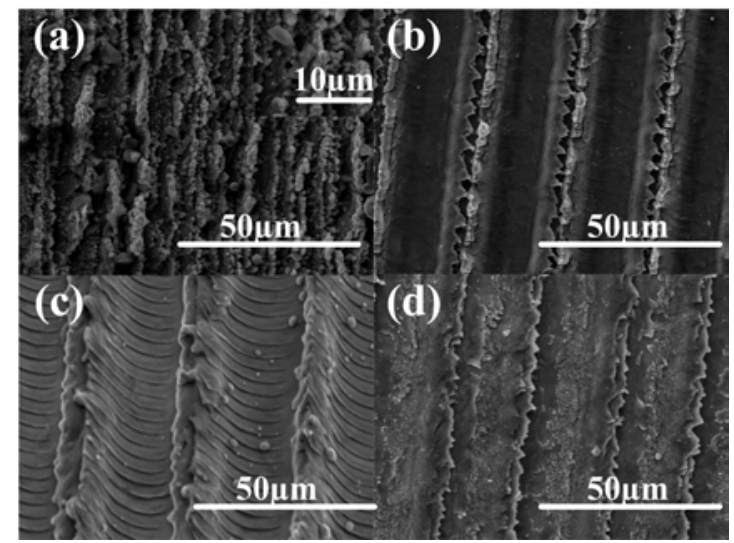

Fig. 4 SEM images of the Ti-Ni memory alloy surface under different laser scanning speed, (a) $10 \mathrm{~mm} / \mathrm{s}$, (b) $30 \mathrm{~mm} / \mathrm{s}$, (c) 50 $\mathrm{mm} / \mathrm{s}$, (d) $80 \mathrm{~mm} / \mathrm{s}$.

The formation mechanism of this unique fish scale-like microstructures has not been thoroughly researched. The schematic diagram of fish-scale like structures forming at the bottom of trench can be seen in Fig. 4. This maybe owing to the following reasons: firstly, the faster scanning speed means the higher laser spot repetition rate. The laser repetition rate is $90 \%$ when the scanning speed is about 10 $\mathrm{mm} / \mathrm{s}$. The laser repetition rate decreases to $80 \%$ when the scanning speed increases to $50 \mathrm{~mm} / \mathrm{s}$. The theoretical distance between two spots is about $7.28 \mu \mathrm{m}$, which is consistent with the distance shown in Fig. 4 (c). The laser repetition rate is only $60 \%$. In addition, for slower scanning speed, corresponding to higher temperature and longer interaction time, the surface can absorb large amount of heat, which makes strong-ablation fragmentation happening [17] Thus, the redeposited microparticles accumulate enough remelting structure to form uniform distributed microbumps, as shown in Fig. 4 (a). The faster scanning speed, implying less instantaneous absorbed energy and shorter interaction time, leads to the weak-ablation spallation [18].In the suitable laser scanning speed, the interval time is long enough for melting region solidification before the next laser spot irradiation. After serval spots accumulating, the fish scalelike structure would form at the bottom of scanning line.

Roughness plays an important role in determining the surface wettability. The calf serum and glycerol contact angles (CA) and sliding angles (SA) are measured to investigate the wettability of as-prepared Ti-Ni surface. Fig. 2 shows the contact angle and sliding angle of sample processed with different laser fluences from $3.5 \mathrm{~J} / \mathrm{cm}^{2}$ to 28 $\mathrm{J} / \mathrm{cm}^{2}$ at the same scanning speed of $50 \mathrm{~mm} / \mathrm{s}$. It can be clearly seen that both calf serum and glycerol contact angles increase with laser fluence. The SAs reveal to opposite trend. This result is corresponding with the surface roughness. When laser fluence is larger than $20 \mathrm{~J} / \mathrm{cm}^{2}$, the calf serum CA is above $150^{\circ}$ and $\mathrm{SA}$ is less than $10^{\circ}$, which reach the standard of superhydrophobic surface. According to their SEM images, we find that the rougher ridge struc- 
tures combined with fish-scale like microstructures lead to high CA and low SA. For the low laser fluence, the ridge part is flat, the calf serum would wet the ridge surface, leading to relative low CA and high SA. For the high laser fluence, the nano-micro binary structures covers the whole ridge surface. Hence the calf serum is hard to wet the ridge surface, which leads to high CA and low SA. According to the Cassie model [19], nano-micro-binary structures can capture a large amount of air, which is beneficial for forming a composite solid-liquid-vapor interface to realize superhydrophobicity. The apparent contact angle is formulated as follows:

$\cos \theta c=f_{1}\left(1+\cos \theta_{1}\right)-1$, where $\theta c$ is the apparent contact angle of the interface, $\theta_{1}$ is the contact angle of smooth TiNi surface after FAS-17 modification, $f_{1}$ is the area fraction for the solid surface in contact with the liquid droplet. By measuring of surface tension instrument, it is found that it is about $156^{\circ}, \theta_{1}$ is $100^{\circ}$. Therefore, the area fraction can be calculated from equation (1). The solid area fraction for the laser processed Ti-Ni surface was $10.0 \%$, obviously the air in the proportion of the composite contact interface is as high as $90 \%$.

Fig. 5 shows the relationship between calf serum and glycerol contact angles and sliding angles and laser scanning speeds. Varying the scanning speed from $10 \mathrm{~mm} / \mathrm{s}$ to $80 \mathrm{~mm} / \mathrm{s}$, we obtain surfaces with different contact angles and sliding angles. Similarly to the influence of laser fluence, low scanning speed means rougher surface. Thus, calf serum and glycerol contact angles decreases with scanning speed and sliding angles increase with scanning speed. Because the scanning speed can severely impact on the processing efficiency. Although, the surface processed with scanning speed of $10 \mathrm{~mm} / \mathrm{s}$ can research superhydrophobicity. Its processing efficiency is too low to large area fabricating superhydrophobic surface. According to the CA and SA results of Fig. 5, we find that the optimal scanning speed is $50 \mathrm{~mm} / \mathrm{s}$ and laser fluence is $28 \mathrm{~J} / \mathrm{cm}^{2}$, which can balance the superhydrophobicity and processing efficiency.

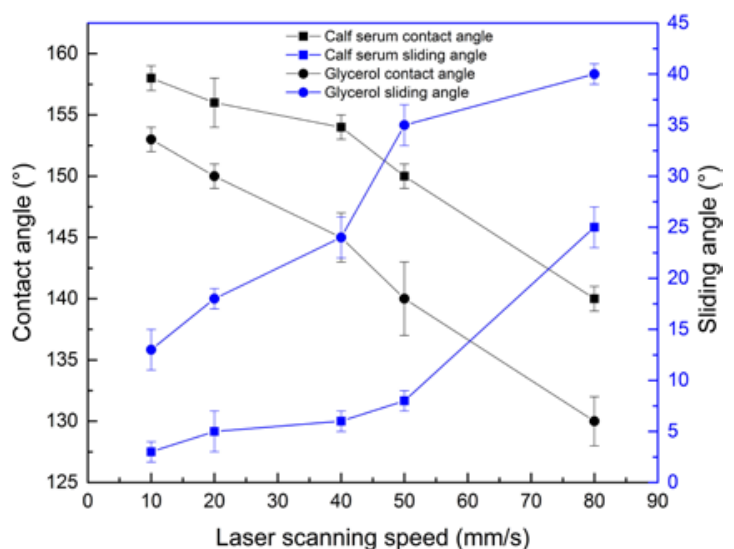

Fig. 5 Contact angles and sliding angles of Ti-Ni alloy surface under different laser scanning speed.

The corrosion may happen when the Ti-Ni alloy implant into tissues. Corrosion resistance of the as-prepared superhydrophobic surface is a key factor in determining the possibilities of the superhydrophobic surface in further medical implant applications. The Tafel polarization curves of original Ti-Ni alloy surface and FAS-17 modified super- hydrophobic Ti-Ni surface are carried out to measure their corrosion potential and corrosion current in $20 \mathrm{wt} \%$ calf serum solution. As shown in Fig. 6 and Table. 1, it can be noted that the superhydrophobic surface has better corrosion resistance than original Ti-Ni alloy surface because it has higher corrosion potential $\left(E_{c o r r}=52 \mathrm{mV}\right)$ and lower corrosion current $\left(I_{\text {corr }}=3.21 \times 10-6 \mathrm{~A} / \mathrm{cm}^{2}\right)$ with comparison of original $\mathrm{Ti}-\mathrm{Ni}$ alloy surface $\left(E_{\text {corr }}=180 \mathrm{mV}\right.$ and $\left.I_{\text {corr }}=9.19 \times 10-6 \mathrm{~A} / \mathrm{cm}^{2}\right)$. The above results show that the superhydrophobic surface has a good corrosion protection to that of original Ti-Ni alloy surface. This maybe due to the fact that the air layer trapped in microstructures can form an air protective layer, which can prevent $\mathrm{NaCl}$ solution penetrating into the micro-nano hierarchical structures and reduce the real contact area between $\mathrm{NaCl}$ solution and microstructures [20].

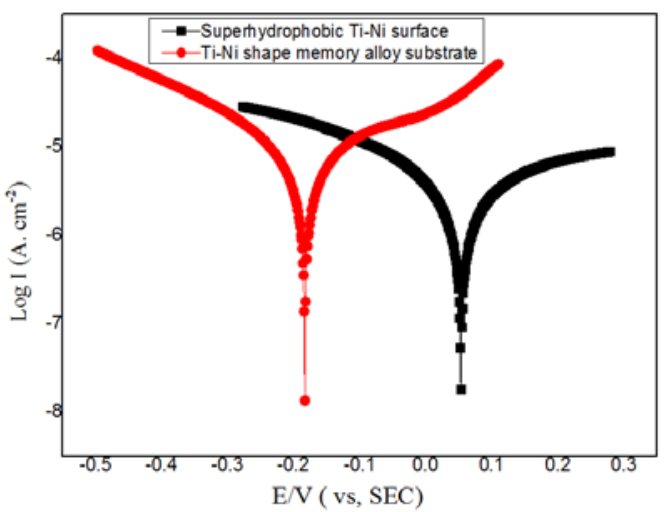

Fig. 6 Tafel polarization curves of Ti-Ni shape memory alloy surface and superhydrophobic Ti-Ni surface.

Table 1 The values of $E_{\text {corr }}$ and $i_{\text {corr }}$

\begin{tabular}{ccc} 
Sample & $E_{\text {corr }}(\mathrm{mV})$ & $i_{\text {corr }}\left(\mathrm{A} / \mathrm{cm}^{2}\right)$ \\
\hline $\begin{array}{c}\text { Ti-Ni shape memory alloy } \\
\text { substrate }\end{array}$ & -180 & $9.19 \times 10^{-6}$ \\
$\begin{array}{c}\text { Superhydrophobic Ti-Ni } \\
\text { shape memory alloy }\end{array}$ & 52 & $3.21 \times 10^{-6}$ \\
\hline
\end{tabular}

Self-cleaning ability is another very important to superhydrophobic surfaces for their practical applications. Figs. 7 (a)-(e) show the self-cleaning effect of the bare Ti-Ni surface and the as-prepared superhydrophobic placed at a fixed slope angle of $5^{\circ}$. Firstly, the original Ti-Ni alloy surface and superhydrophobic are contaminated deliberately with $\mathrm{SiO}_{2}$ powder as dust. Subsequently, the water is dropped to the upper side of the surface by a plastic dropper. When the water droplet slides along superhydrophobic area, the rolling water droplet was able to pick up the dust and slides away from the superhydrophobic area. When the water droplet contacts the original Ti-Ni surface, owing to the intrinsic hydrophilicity of original $\mathrm{Ti}-\mathrm{Ni}$ surface, the water droplet sticks at the surface. The $\mathrm{SiO}_{2}$ dust might adhere to the original $\mathrm{Ti}-\mathrm{Ni}$ surface and cannot be completely removed even under water flushing. This experiment indicates that the as-prepared Ti-Ni superhydrophobic has an excellent lotus-like self-cleaning effect, which can avoid the implant polluted by dust. 


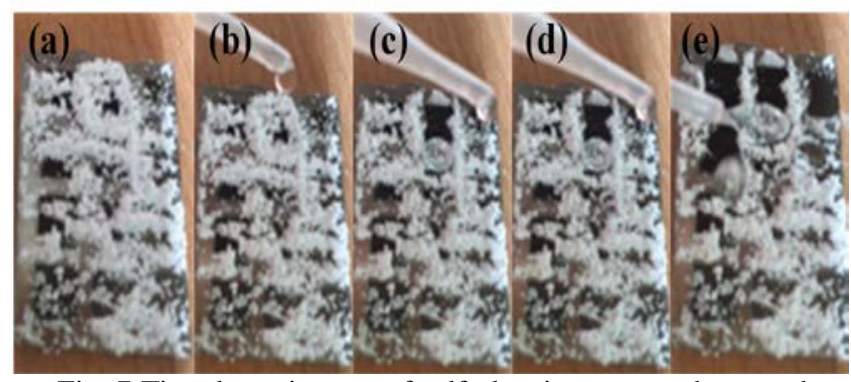

Fig. 7 Time lapse images of self-cleaning test on the superhydrophobic Ti-Ni surface

\section{Conclusions}

By using 355nm ultraviolet nanosecond laser direct writing technology, we prepare superhydrophobic and oleophobic Ti-Ni memory alloy surface. We successfully fabricated hierarchical micro/nano-trench textures composed with fish scale-like microstructures at the bottom and spherical microprotuberance covered by hairy nanostructures on the ridge when the laser fluence is $20 \mathrm{~J} / \mathrm{cm}^{2}$ and laser scanning speed is $50 \mathrm{~mm} / \mathrm{s}$. This unique hierarchical micro/nano trench textures bring an excellent superhydrophobicity with the calf serum contact angles (CA) of $156^{\circ}$ and sliding angles (SA) less than $5^{\circ}$, meanwhile, the surface shows oleophobicity with glycerol contact angles (CA) of $130^{\circ}$ and sliding angles (SA) of $30^{\circ}$. In addition to excellent superhydrophobicity and oleophobicity, this surface also has anticorrosion and self-cleaning ability. Furthermore, we believe that this kind of superhydrophobic Ti$\mathrm{Ni}$ alloy surface would have some potential applications in medical implant, such as heart stents and teeth pins.

\section{Acknowledgements}

This work was supported by Fund for the Frontier Research of the Discipline (2017XKQY001) and A Project Funded by the Priority Academic Program Development of Jiangsu Higher Education Institutions.

\section{References}

[1] B. Bhushan, Y. C. Jung, A. Niemietz and K. Koch: Langmuir, 25, (2009) 1659.

[2] Y. Zheng, X. Gao and L. Jiang: Soft Matter, 3, (2007) 178.

[3] L. Feng, S. Li, Y. Li, H. Li, L. Zhang, J. Zhai, Y. Song, B. Liu, L. Jiang and D. Zhu: Adv. Mater., 14, (2002) 1857.

[4] X. Gao and L. Jiang, Nature, 432, (2004) 36.

[5] H. Liu, X. Wang and H. Ji: Appl. Surf. Sci, 288, (2014) 341.

[6] Y. Tang, Q. Zhang, X. Zhan and F. Chen: Soft Matter, 11, (2015) 4540.

[7] J. Li, L. Yan, H. Li, J. Li, F. Zha and Z. Lei: Rsc. Adv., 5, (2015) 53802.

[8] S. Rezaei, I. Manoucheri, R. Moradian and B. Pourabbas: Chem. Eng. J, 252, (2014) 11.

[9] M. Tang, M. H. Hong, and Y. S. Choo: Appl. Phys. AMater., 101, (2010) 503.

[10] M. Tang, V. Shim, Z. Y. Pan, Y. S. Choo and M. H. Hong: J. Laser Micro/Nanoengin., 6, (2010), 6.

[11] Huangping Yan, Mohamed Raiz B Abdul Rashid, Si Ying Khew, Fengping Li, Minghui Hong: Opto-Electronic Engineering, 44, (2017), 587.
[12] Saltuganov P N, Ionin A A, Kudryashov S I: J. Russ Laser Res., 36, (2015) 81.

[13] M. Gubko, A. Ionin, S. Kudryashov, S. Makarov, A. Rudenko, L. Seleznev and D. Sinitsyn: JETP Lett., 97, (2013) 599.

[14] N. Bloembergen: IEEE. J. Quantum Elect., 10, (1974) 375.

[15] A. A. Ionin, S. I. Kudryashov: J. Exp. Theor. Phys., 116, (2013) 347.

[16] J. Kido, H. Hayase, K. Hongawa, K. Nagai and K. Okuyama: Appl. Phys. Lett., 65, (1994) 2124.

[17] A. A. Ionin, S. I. Kudryashov, A. E. Ligachev: JETP Lett., 94, (2011) 266.

[18] Kudryashov S I, Golosov E V, Ionin A A: Proc. SPIE, 7719, (2010) 771921.

[19] A. Cassie and S. Baxter: Trans. Faraday. Soc., 40, (1944) 546.

[20] J. Ou, W. Hu, M. Xue, F. Wang and W. Li: Acs Appl. Mater. Inter., 5, (2013) 3101.

(Received: August 3, 2017, Accepted: October 12, 2017) 